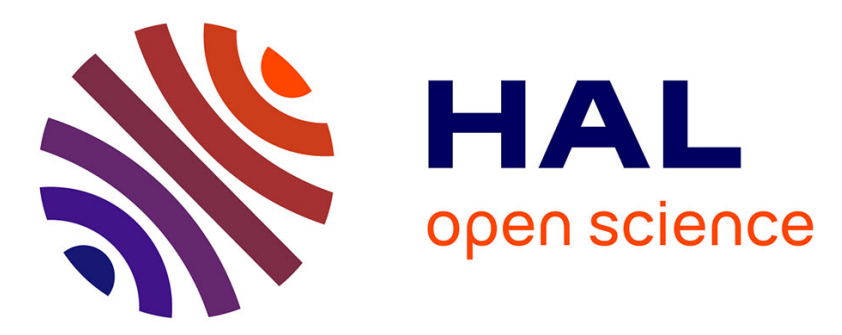

\title{
Regional Stability Analysis of Discrete-Time Dynamic Output Feedback Under Aperiodic Sampling and Input Saturation
}

João Manoel Gomes da Silva, Isabelle Queinnec, Alexandre Seuret, Sophie Tarbouriech

\section{To cite this version:}

João Manoel Gomes da Silva, Isabelle Queinnec, Alexandre Seuret, Sophie Tarbouriech. Regional Stability Analysis of Discrete-Time Dynamic Output Feedback Under Aperiodic Sampling and Input Saturation. IEEE Transactions on Automatic Control, 2016, 61 (12), pp.4176 - 4182. 10.1109/TAC.2016.2553365 . hal-01474407

\section{HAL Id: hal-01474407 \\ https://hal.science/hal-01474407}

Submitted on 22 Feb 2017

HAL is a multi-disciplinary open access archive for the deposit and dissemination of scientific research documents, whether they are published or not. The documents may come from teaching and research institutions in France or abroad, or from public or private research centers.
L'archive ouverte pluridisciplinaire HAL, est destinée au dépôt et à la diffusion de documents scientifiques de niveau recherche, publiés ou non, émanant des établissements d'enseignement et de recherche français ou étrangers, des laboratoires publics ou privés. 


\section{Regional stability analysis of discrete-time dynamic output feedback under aperiodic sampling and input saturation*}

João Manoel Gomes da Silva Jr., Isabelle Queinnec, Alexandre Seuret, Sophie Tarbouriech

\begin{abstract}
This paper addresses the problem of regional stability analysis of a continuous-time plant controlled by a discrete-time dynamic output feedback control law with saturation constraints under aperiodic sampling. To cope with the aperiodic sampling problem, the proposed approach is based on an impulsive system modeling and the use of looped functionals. A generalized sector-based relation is applied to tackle the control saturation effects. From these ingredients, conditions to ensure regional asymptotic stability of the closed-loop system under aperiodic sampling are derived. Based on these conditions, LMI-based optimization problems are proposed to compute estimates of the region of attraction of the closed-loop system or to maximize the bound on the maximal admissible interval between two successive sampling instants, for which the regional stability can be ensured with respect to a given set of admissible states. A numerical example illustrates the application of the results.
\end{abstract}

Keywords. Dynamic output feedback, saturating input, sampled-data systems, aperiodic sampling.

\section{INTRODUCTION}

Issues regarding the implementation of sampled-data systems and the effects of sampling on the stability of closed-loop systems have been intensively studied since the 80 's (see, for instance, [1], [5], [6], [17], [23], [22]). More recently, mainly motivated by networked control applications [31], stability of aperiodic sampled-data systems has benefited from a second wave of theoretical developments. The results in this context allow to account for aperiodic samplings in a formal way and basically rely upon four efficient approaches of modeling. The first one is based on an uncertain discrete-time model, which is embedded onto a polytopic model from the use of exponential matrices [7], [8]. A second approach refers to a robust analysis of continuous or discrete-time systems. The effects of the aperiodic sampling is embedded into norm bounded uncertainties and the proposed results are based on region-dividing techniques of the admissible inter sampling interval [12] or on IQCs and passivity arguments [20], [24]. Another approach regards the modeling of the sampling effects considering a particular time-varying delay on the control signal. This approach, initially proposed in [11] and further improved in [10] or [21] is based on the use of Lyapunov-Krasovskii functionals and impulsive systems representation for systems with time-varying delays. Similarly to [28], a mixed continuous- and discrete-time approach has been proposed in [26], [27] through the concept of looped-functionals and adapted to the case of impulsive systems in [4]. This alternative method authorizes a larger class of functionals than in the Lyapunov-Krasovskii approach and has some similarities with the notion of clock-dependent Lyapunov-function, employed for instance in [2]. The reader may refer to [3] for detailed comparison between these two approaches. It should however be highlighted that the aforementioned methods are restricted to state feedback control laws.

*This work was supported by ANR-France projects LimICoS (contract 12 BS03 005 01) and SCIDiS (contract 15-CE23-0014), STICAmSud project ADNEC, CNPq-Brazil (grants Univ. 480638/2012-8 and PQ 305979/2015-9) and CAPES-Brazil (projects PVE-129/2012 and STICAmSud)

J.M. Gomes da Silva Jr. is with UFRGS, EE, Department of Automation and Energy (DELAE), Porto Alegre, Brazil jmgomes@ece.ufrgs.br

I. Queinnec, A. Seuret and S. Tarbouriech are with CNRS, LAAS, 7 av. du Colonel Roche, F-31400 Toulouse, France and with Univ de Toulouse, LAAS, F-31400 Toulouse, France queinnec, aseuret, tarbour@laas.fr
On the other hand, a large attention has been paid to the stability analysis and stabilization of systems with input saturation (see, for example, [19], [29]). In this context, many methods guaranteeing global or regional (local) asymptotic stability of the closed-loop systems under saturating control laws have been proposed, either in the continuous or discrete-time frameworks. Considering the periodic sampling case, the problem of assessing stability of a continuoustime plant controlled by a discrete-time dynamic output feedback control law has been addressed for instance, in [9]. In [18], the authors extended the design of discrete-time anti-windup loops to the sampled-data case with a constant period by using the approach proposed in [5], in order to transpose the problem into a discrete-time framework.

In the present paper, we are interested in analyzing stability of a continuous-time plant controlled by a discrete-time dynamic output feedback control law with saturation constraints under aperiodic sampling. In this context, to cope with the aperiodic sampling problem, the proposed approach is based on the impulsive system modeling as suggested in [13] and on the use of looped functionals. Furthermore, a generalized sector-based condition is applied to tackle the control saturation effects. From these ingredients, conditions to ensure regional asymptotic stability of the closed-loop system under aperiodic sampling are derived. Based on these conditions, LMIbased optimization problems are proposed to compute estimates of the region of attraction of the closed-loop system or to maximize the bound on the maximal admissible interval between two successive sampling instants, for which regional stability can be ensured for a given set of admissible states.

Notation. $\mathbb{S}^{n}$ denote the set symmetric matrices of $\mathbb{R}^{n \times n}$. For given positive scalars $0<\mathcal{T}_{1} \leq \mathcal{T}_{2}, \mathbb{K}$ is defined as the set of continuous functions from an interval $[0, T]$ to $\mathbb{R}^{n}$, where $T$ is a positive scalar in $\left[\mathcal{T}_{1}, \mathcal{T}_{2}\right] .|\cdot|$ and $\|\cdot\|$ stand for the absolute value and the Euclidean norm, respectively. The notation $P>0$ for $P \in \mathbb{S}^{n}$ means that $P$ is a symmetric positive definite matrix. For any positive integer $j \leq n$, any vector $x \in \mathbb{R}^{n}$ and any matrix $A \in \mathbb{R}^{n \times m}, A_{j}, x_{j}$ refer to the $j$ th line of matrix $A$, the $j$ th component of vector $x$, respectively. For $A \in R^{n \times m}$, $\mathrm{He}\{A\}=A+A^{\prime} . I$ and 0 represent the identity and zero matrices of appropriate dimensions. $C o\{\cdot\}$ denotes a convex hull. For $v \in \mathbb{R}^{m}$, each component of the vector-valued saturation function, $\operatorname{sat}(v)$, is defined by $\operatorname{sat}(v)_{i}=\operatorname{sign}\left(v_{i}\right) \min \left\{u_{0 i},\left|v_{i}\right|\right\}, \quad i=1, \ldots, m$, where $\pm u_{0 i}$ denote the symmetric limits on the $i$ th component of $v$.

\section{PROBLEM FORMULATION}

\section{A. Plant model}

Consider the continuous-time linear plant with saturating inputs described by:

$$
\left\{\begin{array}{l}
\dot{x}_{p}(t)=A_{p} x_{p}(t)+B_{p} \operatorname{sat}(\bar{u}(t)), \\
y(t)=C_{p} x_{p}(t),
\end{array}\right.
$$

where $x_{p} \in \mathbb{R}^{n_{p}}, \bar{u} \in \mathbb{R}^{m}$ and $y \in \mathbb{R}^{p}$ represent the state, the input and the output vectors of the plant, respectively. Matrices $A_{p}, B_{p}$, $C_{p}$ have appropriate dimensions and are supposed to be constant. We assume that the control inputs are sampled, possibly in an aperiodic manner, and the value of $\bar{u}$ is kept constant (through a zero-order hold) between two successive sampling instants i.e.:

$$
\bar{u}(t)=u\left(t_{k}\right), \quad \forall t \in\left[t_{k}, t_{k+1}\right),
$$

The sequence of increasing positive sampling instants is denoted by $\mathbb{T}:=\left\{t_{k}\right\}_{k \geq 0}$. It is assumed that $\mathbb{T}:=\left\{t_{k}\right\}_{k \geq 0}$ is such that $\bigcup_{k \in \mathbb{N}}\left[t_{k}, t_{k+1}\right)=[0,+\infty)$, so that no Zeno phenomena occurs. Moreover, we assume that there exist two positive scalars $\mathcal{T}_{1} \leq \mathcal{T}_{2}$ 
such that the difference between two successive sampling instants $T_{k}=t_{k+1}-t_{k}$ satisfies

$$
0<T_{1} \leq T_{k} \leq \mathcal{T}_{2}, \quad \forall k \in \mathbb{N} .
$$

Note that the inter sampling time $T_{k}$ can vary with time, which allows to model an aperiodic sampling policy. The particular case of periodic sampling corresponds to $T_{k}=\mathcal{T}_{1}=\mathcal{T}_{2}=\delta, \forall k \in \mathbb{N}$.

For output feedback purposes, we consider that the output $y(t)$ is sampled at the same instants of the control input, i.e. at each sampling instant $t_{k}$, the sample $y\left(t_{k}\right)$ is generated.

\section{B. Discrete-time dynamic output feedback controller}

Considering a digital implementation, we assume that system (1) is controlled by a linear discrete-time dynamic output feedback controller, described in state space as follows:

$$
\begin{aligned}
& x_{c}\left(t_{k+1}\right)=A_{c} x_{c}\left(t_{k}\right)+B_{c} y\left(t_{k}\right)+E_{c} \psi\left(u\left(t_{k}\right)\right), \\
& u\left(t_{k}\right)=C_{c} x_{c}\left(t_{k}\right)+D_{c} y\left(t_{k}\right),
\end{aligned}
$$

where $x_{c} \in \mathbb{R}^{n_{c}}, y \in \mathbb{R}^{p}$ and $u \in \mathbb{R}^{m}$ are the state, the input and the output of the controller, respectively. The matrices $A_{c}, B_{c}, C_{c}$, $D_{c}, E_{c}$ are assumed to be constant and of appropriate dimensions. $\psi(u)$ is a vector-valued decentralized deadzone nonlinearity defined as follows:

$$
\psi(u)=\operatorname{sat}(u)-u .
$$

The term $E_{c} \psi(u)$ regards a static anti-windup compensation, which can be appended to the controller to mitigate the saturation effects on performance and stability [15], [16].

In this case, at each sampling instant, the plant output is sampled and its value is used to instantaneously update both the controller state and the control input to be applied to the plant between in the interval $\left[\begin{array}{ll}t_{k} & t_{k+1}\end{array}\right)$.

Remark 1: It should be noticed that the method used to design the controller (4) is out of the scope of the present work. Our main goal is to provide a method to assess the stability of the sampled-data closed-loop system defined by the connection between this controller and the continuous plant (1). On the other hand, it is reasonable (and relevant from a practical point of view) to assume that (4) has been designed considering classical techniques based on a periodic sampling paradigm, i.e. with $T_{k}=\delta, \forall k$, and then to analyze the effects on stability of a possibly aperiodic sampling policy with $T_{k} \in$ $\left[\mathcal{T}_{1}, \mathcal{T}_{2}\right]$. In this case, we can assume for instance that matrices $A_{c}$, $B_{c}, C_{c}, D_{c}, E_{c}$, have been designed considering one of the two approaches below:

1) Discrete-time design. First, an exact discretization of the plant should be performed. Considering $t_{k+1}-t_{k}=\delta, \forall k$, this leads to the following discrete-time model:

$$
\begin{aligned}
& x_{p}\left(t_{k+1}\right)=A_{p, d} x_{p}\left(t_{k}\right)+B_{p, d} \operatorname{sat}\left(u\left(t_{k}\right)\right), \\
& y\left(t_{k}\right)=C_{p, d} x_{p}\left(t_{k}\right)
\end{aligned}
$$

with $A_{p, d}=e^{A_{p} \delta}, C_{p, d}=C_{p}, B_{p, d}=\int_{0}^{\delta} e^{A_{p}(\delta-s)} d s B_{p}$. Thus, based on model (6), known discrete-time results can be applied to design matrices $A_{c}, B_{c}, C_{c}$ and $E_{c}$ in order to stabilize (considering periodic sampling) the closed-loop system.

2) Controller discretization. First, a continuous-time stabilizing controller, described by

$$
\begin{aligned}
& \dot{x}_{c}(t)=\bar{A}_{c} x_{c}(t)+\bar{B}_{c} y(t)+\bar{E}_{c} \psi(u(t)), \\
& u(t)=\bar{C}_{c} x_{c}(t)+\bar{D}_{c} y(t),
\end{aligned}
$$

is designed considering the continuous-time plant model (1). Then, assuming a periodic sampling, a discretization of this controller can be performed using classical Euler or Tustin approximations. Alternatively, a discrete-time approximation ensuring that the value of the output of the continuous and the discrete-time controllers coincide at the sampling instants $t_{k}=k \delta$ can be considered. This leads to a discrete-time controller as in (4) with:

$$
\begin{aligned}
& A_{c}=e^{\bar{A}_{c} \delta}, \quad C_{c}=\bar{C}_{c}, \quad D_{c}=\bar{D}_{c} \\
& B_{c}=\int_{0}^{\delta} e^{\bar{A}_{c}(\delta-s)} d s \bar{B}_{c}, \quad E_{c}=\int_{0}^{\delta} e^{\bar{A}_{c}(\delta-s)} d s \bar{E}_{c}
\end{aligned}
$$

\section{Hybrid modeling of the closed-loop system}

Considering the controller (4), the state of the plant evolves in continuous-time while the controller state and the plant input have discrete-time updates. These behaviors can be cast in the hybrid dynamical systems framework [13]. From the previous assumptions, the closed-loop system dynamics can be represented by an impulsive system described by:

$$
\begin{aligned}
& \begin{cases}\dot{x}_{p}=A_{p} x_{p}+B_{p} D_{c} C_{p} x_{m}+B_{p} C_{c} x_{c}+B_{p} \psi, & \\
\dot{x}_{m}=0, & \forall t \in \mathbb{R} \backslash \mathbb{T}, \\
\dot{x}_{c}=0, & \forall t \in \mathbb{T},\end{cases} \\
& \begin{cases}x_{p}^{+}=x_{p}, \\
x_{m}^{+}=x_{p}, \\
x_{c}^{+}=A_{c} x_{c}+B_{c} C_{p} x_{m}+E_{c} \psi,\end{cases}
\end{aligned}
$$

where $x^{+}=x\left(t_{k+1}\right)$ and $\psi$ is the deadzone function given by

$$
\psi=\operatorname{sat}\left(C_{c} x_{c}+D_{c} C_{p} x_{m}\right)-\left(C_{c} x_{c}+D_{c} C_{p} x_{m}\right)
$$

When $t \in \mathbb{R} \backslash \mathbb{T}$, the evolution of the plant state follows the first differential equation. The two other variables, representing the held values of the plant state and the controller state, remain constant in the interval $\left[t_{k}, t_{k+1}\right)$. Since the plant dynamics evolves continuously, if $t \in \mathbb{T}$ the plant state remains at its current value (i.e. $x_{p}^{+}=x_{p}$ ), while the other variables are impulsively reset. We introduced the auxiliary variable $x_{m}$ in (9) to represent the memory of the current sampled value of the plant state. Actually, it couples the continuous and the discrete-time dynamics through the control signal. Note that $u\left(t_{k}\right)=C_{c} x_{c}\left(t_{k}\right)+D_{c} y\left(t_{k}\right)=C_{c} x_{c}\left(t_{k}\right)+D_{c} C_{p} x_{m}\left(t_{k}\right)$.

\section{Stability Analysis Problems}

Considering the nonlinear closed-loop system (9) we are interested in analyzing both the influence of the aperiodic sampling and the control saturation on its asymptotic stability. Since no particular assumption is assumed regarding the stability of the continuous-time plant, i.e. matrix $A_{p}$ is not necessarily Hurwitz, we mainly focus on the regional stability analysis. Actually, it should be recalled that if the open-loop system is exponentially unstable, only regional stability can be ensured in the presence of control saturation [29]. In this case, the region of attraction of the origin is defined as the set of all initial states for which the corresponding trajectories converge asymptotically to the origin. Nevertheless, the exact characterization of this region is in general not possible. Hence, it is of major interest the determination of analytical estimates of the region of attraction, which can be seen as regions of asymptotic stability (RAS) [29]. Hence, we are concerned by the following problems:

P1. Given $\mathcal{T}_{1}$ and $\mathcal{T}_{2}$, such that $0<\mathcal{T}_{1} \leq \mathcal{T}_{2}$, determine an estimate of the region of attraction of system (9).

P2. Given a set of admissible initial states $\mathcal{X}_{0}$, maximize the length of the interval $\left[\mathcal{T}_{1}, \mathcal{T}_{2}\right]$ of admissible inter sampling times $T_{k}$, for which asymptotic stability of system (9) is ensured for any initial condition in $\mathcal{X}_{0}$. 


\section{PRELIMINARIES}

\section{A. Looped-functional approach}

In order to tackle problems P1 and P2, instead of considering the classical hybrid framework to study mixed continuous and discrete dynamics as defined in [13], we use an alternative direction by adapting the technique based on a looped functional as developed in [4]. This approach relies on the characterization of the trajectories of system (9) in a lifted domain [30], [4], [26]. The idea is to view the entire state-trajectory as a sequence of lifted state function $\chi_{k}(\tau) \in \mathbb{K}, \chi_{k}:\left[0, T_{k}\right] \rightarrow \Re^{n}$, with $\left[0, T_{k}\right] \subset\left[0, \mathcal{T}_{2}\right]$ and the vector $\chi_{k}(\tau) \in \mathbb{R}^{n}$, with $n=2 n_{p}+n_{c}$, being defined as follows:

$$
\begin{gathered}
\chi_{k}(\tau)=\left[\begin{array}{l}
\chi_{p, k}(\tau) \\
\chi_{m, k}(\tau) \\
\chi_{c, k}(\tau)
\end{array}\right]=\left[\begin{array}{l}
x_{p}\left(t_{k}+\tau\right) \\
x_{m}\left(t_{k}+\tau\right) \\
x_{c}\left(t_{k}+\tau\right)
\end{array}\right] \\
\text { with } \quad \chi_{k}(0)=\lim _{s \rightarrow t_{k}^{+}}\left[\begin{array}{l}
x_{p}(s) \\
x_{m}(s) \\
x_{c}(s)
\end{array}\right] .
\end{gathered}
$$

Then, by using (10), the hybrid closed-loop system (9) can be alternatively represented as follows:

$$
\left\{\begin{aligned}
\dot{\chi}_{p, k}(\tau)= & A_{p} \chi_{p, k}(\tau)+B_{p} D_{c} C_{p} \chi_{m, k}(\tau) \\
& +B_{p} C_{c} \chi_{c, k}(\tau)+B_{p} \psi_{k}(\tau), \\
\dot{\chi}_{m, k}(\tau)= & 0 \\
\dot{\chi}_{c, k}(\tau)= & 0, \\
\chi_{p, k+1}(0)= & \chi_{p, k}\left(T_{k}\right), \\
\chi_{m, k+1}(0)= & \chi_{p, k}\left(T_{k}\right), \\
\chi_{c, k+1}(0)= & A_{c} \chi_{c, k}\left(T_{k}\right)+B_{c} C_{p} \chi_{m, k}\left(T_{k}\right) \\
& +E_{c} \psi_{k}\left(T_{k}\right),
\end{aligned}\right.
$$

Note that, since $\dot{\chi}_{m, k}(\tau)=0$ and $\dot{\chi}_{c, k}(\tau)=0$, it follows that $\dot{\psi}_{k}(\tau)=0$, i.e. $\psi_{k}(\tau)=\psi_{k}(0)=\psi\left(C_{c} \chi_{c, k}(0)+D_{c} C_{p} \chi_{m, k}(0)\right)$, for all $\tau \in\left[0, T_{k}\right]$. Moreover $\chi_{m, k}\left(T_{k}\right)=\chi_{m, k}(0)$ and $\chi_{c, k}\left(T_{k}\right)=$ $\chi_{c, k}(0)$.

Based on the lifted function $\chi_{k}$, we recall the following result, which is the basis of the looped-functional approach to assess asymptotic stability of system (9).

Lemma 1: [4] Consider system (9) and let $0<\mathcal{T}_{1} \leq \mathcal{T}_{2}$, be two positive scalars and $V: \mathbb{R}^{n} \rightarrow \mathbb{R}^{+}$be a function for which there exist real scalars $0<\mu_{1}<\mu_{2}$ such that

$$
\forall \chi \in \mathbb{R}^{n}, \quad \mu_{1}\|\chi\|^{2} \leq V(\chi) \leq \mu_{2}\|\chi\|^{2} .
$$

Then, the two following statements are equivalent.

(i) For all $k \in \mathbb{N}, T_{k} \in\left[\mathcal{T}_{1}, \mathcal{T}_{2}\right]$, the forward difference of the function $V$ satisfies

$$
\Delta V(k):=V\left(\chi_{k+1}(0)\right)-V\left(\chi_{k}(0)\right)<0 ;
$$

(ii) There exists a continuous functional $\mathcal{V}_{0}:\left[0, \mathcal{T}_{2}\right] \times \mathbb{K} \rightarrow \mathbb{R}$, which satisfies, for all $z \in \mathbb{K}$ and $\forall T_{k} \in\left[\mathcal{T}_{1}, \mathcal{T}_{2}\right]$,

$$
\mathcal{V}_{0}\left(T_{k}, z\right)=\mathcal{V}_{0}(0, z)
$$

and such that, for all $k \in \mathbb{N}, T_{k} \in\left[\mathcal{T}_{1}, \mathcal{T}_{2}\right]$ and $\tau \in\left[0, T_{k}\right]$,

$$
\dot{\mathcal{W}}\left(\tau, \chi_{k}\right)=\frac{\Lambda_{k}}{T_{k}}+\frac{d}{d \tau}\left[V\left(\chi_{k}(\tau)\right)+\mathcal{V}_{0}\left(\tau, \chi_{k}\right)\right]<0,
$$

with $\Lambda_{k}=V\left(\chi_{k+1}(0)\right)-V\left(\chi_{k}\left(T_{k}\right)\right)$.

Moreover, if one of these two statements holds, then system (9) is asymptotically stable for all $T_{k} \in\left[\mathcal{T}_{1}, \mathcal{T}_{2}\right]$.
Note that from this result it suffices to analyze the behavior of the system in the interval $\left[0, T_{k}\right]$ to assess asymptotic stability of the closed-loop system (9). Moreover, differently from other approaches based on Lyapunov functionals, an important feature of the present approach is that $\mathcal{V}_{0}$ is not required to be positive.

\section{B. Generalized sector condition}

Based on the variables used to compute the control signal, let us define $\bar{\chi}_{k}=\left[\begin{array}{l}\chi_{m, k} \\ \chi_{c, k}\end{array}\right]$. From this definition it follows that $\psi_{k}(0)=$ $\psi_{k}\left(K \bar{\chi}_{k}(0)\right)$ with $K=\left[\begin{array}{ll}D_{c} C_{p} & C_{c}\end{array}\right]$.

Thus, the effects of the saturation nonlinearity can be considered through a generalized sector condition, that applies to deadzone nonlinearities, which is summarized in the next lemma.

Lemma 2: [15], [29] Consider $K, G \in \mathbb{R}^{m \times\left(n_{p}+n_{c}\right)}$ and define the set $\mathcal{S}=\left\{\bar{\chi} \in \mathbb{R}^{n_{p}+n_{c}} ;\left|\left(K_{j}-G_{j}\right) \bar{\chi}\right| \leq u_{0 j}, j=1, \ldots, m\right\}$. If $\bar{\chi} \in \mathcal{S}$, then the deadzone nonlinearity $\psi(K \bar{\chi})$, satisfies the following condition:

$$
\psi(K \bar{\chi})^{\prime} U(\psi(K \bar{\chi})+G \bar{\chi}) \leq 0,
$$

for any diagonal positive definite matrix $U \in \mathbb{R}^{m \times m}$.

\section{MAIN RESUlT}

Based on the two previous Lemmas, the following theorem is stated. It provides conditions to determine an ellipsoidal RAS for system (9) under aperiodic sampling.

Theorem 1: For given positive scalars $0<\mathcal{T}_{1} \leq \mathcal{T}_{2}$, assume that there exist symmetric positive definite matrices $P, R$, symmetric matrices $X, S_{1}$, a diagonal positive definite matrix $U$, matrices $G$, $V, S_{2}$ and $N$ of appropriate dimensions and a positive scalar $\alpha$, that satisfy, for $i=1,2, j=1, \cdots, m$

$$
\begin{aligned}
& \Psi_{1}\left(\mathcal{T}_{i}\right)=\left[\begin{array}{cc}
\Pi_{1}+\mathcal{T}_{i}\left(\Pi_{3}+\Pi_{4}\right) & \mathcal{T}_{i} N \\
\mathcal{T}_{i} N^{\prime} & -\mathcal{T}_{i} R
\end{array}\right]<0, \\
& \Psi_{2}\left(\mathcal{T}_{i}\right)=\Pi_{1}+\mathcal{T}_{i}\left(\Pi_{2}-\Pi_{3}+\Pi_{4}\right)<0, \\
& {\left[\begin{array}{cc}
P+V M_{f}+M_{f}^{\prime} V^{\prime} & {\left[\begin{array}{cc}
0 & K_{j}-G_{j}
\end{array}\right]^{\prime}} \\
{[0} & K_{j}-G_{j}
\end{array}\right]>0,}
\end{aligned}
$$

with

$$
\begin{aligned}
\Pi_{1}= & M_{+}^{\prime} P M_{+}-M_{-}^{\prime} P M_{-}+\Pi_{10} \\
& -\operatorname{He}\left\{M_{5}^{\prime} U M_{5}+M_{5}^{\prime} U G\left[\begin{array}{c}
M_{3} \\
M_{4}
\end{array}\right]\right\}, \\
\Pi_{10}= & M_{12}^{\prime} S_{1} M_{12}+\operatorname{He}\left\{M_{12}^{\prime} S_{2} M_{2}+N M_{12}\right\}, \\
\Pi_{2}= & M_{c}^{\prime} R M_{c}+\operatorname{He}\left\{M_{c}^{\prime}\left(S_{1} M_{12}+S_{2} M_{2}\right)\right\}, \\
\Pi_{3}= & {\left[\begin{array}{c}
M_{2} \\
M_{5}
\end{array}\right]^{\prime} X\left[\begin{array}{c}
M_{2} \\
M_{5}
\end{array}\right], } \\
\Pi_{4}= & \operatorname{He}\left\{\left[\begin{array}{c}
M_{c} \\
0 \\
0
\end{array}\right]^{\prime} P\left[\begin{array}{l}
M_{1} \\
M_{3} \\
M_{4}
\end{array}\right]\right\},
\end{aligned}
$$

where

$$
\begin{aligned}
& M_{1}=\left[\begin{array}{lllll}
I & 0 & 0 & 0 & 0
\end{array}\right], M_{2}=\left[\begin{array}{lllll}
0 & I & 0 & 0 & 0
\end{array}\right], \\
& M_{3}=\left[\begin{array}{lllll}
0 & 0 & I & 0 & 0
\end{array}\right], M_{4}=\left[\begin{array}{lllll}
0 & 0 & 0 & I & 0
\end{array}\right], \\
& M_{5}=\left[\begin{array}{lllll}
0 & 0 & 0 & 0 & I
\end{array}\right], M_{12}=M_{1}-M_{2} \text {, } \\
& M_{+}=\left[\begin{array}{ccccc}
0 & I & 0 & 0 & 0 \\
0 & I & 0 & 0 & 0 \\
0 & 0 & B_{c} C_{p} & A_{c} & E_{c}
\end{array}\right], M_{-}=\left[\begin{array}{ccccc}
0 & I & 0 & 0 & 0 \\
0 & 0 & I & 0 & 0 \\
0 & 0 & 0 & I & 0
\end{array}\right] \text {, } \\
& M_{f}=\left[\begin{array}{lll}
I & -I & 0
\end{array}\right], M_{c}=\left[\begin{array}{lllll}
A_{p} & 0 & B_{p} D_{c} C_{p} & B_{p} C_{c} & B_{p}
\end{array}\right],
\end{aligned}
$$


Then, if $\chi_{0}(0) \in \mathcal{E}(P, \alpha)=\left\{\chi \in \Re^{n} ; \chi^{\prime} P \chi \leq \alpha^{-1}\right\}$, then for any aperiodic sampling satisfying (3), $\chi_{k}(0) \rightarrow 0$ as $k \rightarrow \infty$, i.e. $\mathcal{E}(P, \alpha)$ is a RAS for the closed-loop system (9).

Proof. Let a quadratic Lyapunov function candidate be defined, for any $\chi$ in $\mathbb{R}^{n}$, by $V(\chi)=\chi^{\prime} P \chi$, where $P \in \mathbb{S}^{n}, P>0$. Thus, the function $V$ satisfies (12). Consider now a functional $\mathcal{V}_{0}$ defined for any $\tau \in\left[0, T_{k}\right]$, as follows:

$$
\begin{aligned}
\mathcal{V}_{0}\left(\tau, \chi_{k}\right) & =\frac{\tau}{T_{k}}\left(\chi_{p, k}(\tau)-\chi_{p, k}\left(T_{k}\right)\right)^{\prime} S_{1}\left(\chi_{p, k}(\tau)-\chi_{p, k}\left(T_{k}\right)\right) \\
& +2 \frac{\tau}{T_{k}}\left(\chi_{p, k}(\tau)-\chi_{p, k}\left(T_{k}\right)\right)^{\prime} S_{2} \chi_{p, k}\left(T_{k}\right) \\
& +\frac{\left(T_{k}-\tau\right)}{T_{k}} \tau\left[\begin{array}{c}
\chi_{p, k}\left(T_{k}\right) \\
\psi_{k}(0)
\end{array}\right]^{\prime} X\left[\begin{array}{c}
\chi_{p, k}\left(T_{k}\right) \\
\psi_{k}(0)
\end{array}\right] \\
& -\frac{\tau}{T_{k}} \int_{\tau}^{T_{k}} \dot{\chi}_{p, k}^{\prime}(\theta) R \dot{\chi}_{p, k}(\theta) d \theta,
\end{aligned}
$$

with $S_{1} \in \mathbb{S}^{n_{p}}, S_{2} \in \mathbb{R}^{n_{p} \times n_{p}}, X \in \mathbb{S}^{n_{p}+m}, R \in \mathbb{S}^{n_{p}}$ such that $R>0$. Since $\mathcal{V}_{0}\left(\tau, \chi_{k}\right)$ is equal to zero at $\tau=0$ and $\tau=T_{k}$, it satisfies condition (13). Moreover, it is continuous at all sampling instants and differentiable over $\left[0, T_{k}\right)$.

Then, from Lemma 1 , if we prove that $\mathcal{W}\left(\tau, \chi_{k}\right)$ defined from $V\left(\chi_{k}\right)$ and $\mathcal{V}_{0}\left(\tau, \chi_{k}\right)$ above, is such that $\dot{\mathcal{W}}\left(\tau, \chi_{k}\right)<0$ along the trajectories of system (9), we can conclude that $\Delta V(k)<0$, which ensures that the trajectories converge asymptotically to the origin.

From Lemma 2 , if $\bar{\chi}_{k}(0) \in \mathcal{S}$, it follows that

$$
\mathcal{W}_{s}\left(\chi_{k}, \psi_{k}\right):=2 \psi_{k}^{\prime}(0) U\left(\psi_{k}(0)+G\left[\begin{array}{c}
\chi_{m, k}(0) \\
\chi_{c, k}(0)
\end{array}\right]\right) \leq 0,
$$

for any diagonal positive definite matrix $U$. Therefore we can combine (21) and (14) to relax the condition $\dot{\mathcal{W}}\left(\tau, \chi_{k}\right)<0$ in a regional context. With this aim, we define

$$
\dot{\mathcal{W}}_{g}\left(\tau, \chi_{k}\right):=\frac{\Lambda_{k}}{T_{k}}+\dot{V}\left(\chi_{k}(\tau)\right)+\dot{\mathcal{V}}_{0}\left(\tau, \chi_{k}\right)-\frac{\mathcal{W}_{s}\left(\chi_{k}, \psi_{k}\right)}{T_{k}} .
$$

Then, if $\dot{\mathcal{W}}_{g}\left(\tau, \chi_{k}\right)<0$, it follows that $\dot{\mathcal{W}}\left(\tau, \chi_{k}\right)<0$ provided that $\bar{\chi}_{k}(0) \in \mathcal{S}$. In order to obtain an upper bound on the functional $\dot{\mathcal{W}}_{g}\left(\tau, \chi_{k}\right)$, we define the extended vector

$$
\zeta_{k}(\tau)=\left[\chi_{p, k}(\tau)^{\prime} \chi_{p, k}\left(T_{k}\right)^{\prime} \chi_{m, k}(0)^{\prime} \chi_{c, k}(0)^{\prime} \psi_{k}(0)^{\prime}\right]^{\prime},
$$

and we compute each term of $\dot{\mathcal{W}}_{g}\left(\tau, \chi_{k}\right)$ separately. Let us first focus on $\Lambda_{k}=V\left(\chi_{k+1}(0)\right)-V\left(\chi_{k}\left(T_{k}\right)\right)$. From the definition of $V\left(\chi_{k}\right)$ and matrices $M_{+}$and $M_{-}$in (20), it follows that

$$
\Lambda_{k}=\zeta_{k}(\tau)^{\prime}\left[M_{+}^{\prime} P M_{+}-M_{-}^{\prime} P M_{-}\right] \zeta_{k}(\tau) .
$$

To compute the second term of $\dot{\mathcal{W}}_{g}, \dot{V}\left(\chi_{k}(\tau)\right)$, note that $\chi_{m, k}(\tau)=\chi_{m, k}(0), \chi_{c, k}(\tau)=\chi_{c, k}(0)$ and $\psi_{k}(\tau)=\psi_{k}(0)$. Hence, considering the auxiliary matrices defined in (20), we get

$$
\frac{d V\left(\chi_{k}(\tau)\right)}{d \tau}=\zeta_{k}(\tau)^{\prime}\left[\operatorname{He}\left\{\left[\begin{array}{c}
M_{c} \\
0 \\
0
\end{array}\right]^{\prime} P\left[\begin{array}{l}
M_{1} \\
M_{3} \\
M_{4}
\end{array}\right]\right\}\right] \zeta_{k}(\tau)
$$

Similarly, the sector condition, $\mathcal{W}_{s}$, can be re-written as follows:

$$
\mathcal{W}_{s}=\zeta_{k}(\tau)^{\prime}\left[\operatorname{He}\left\{M_{5}^{\prime} U\left(M_{5}+G\left[\begin{array}{l}
M_{3} \\
M_{4}
\end{array}\right]\right)\right\}\right] \zeta_{k}(\tau)
$$

Let us finally consider the third component of $\dot{\mathcal{W}}_{g}$, i.e. $\dot{\mathcal{V}}_{0}\left(\tau, \chi_{k}\right)$. Considering the derivative of $\mathcal{V}_{0}$ with respect to $\tau$ and using the matrices $\Pi_{10}, \Pi_{2}$ and $\Pi_{3}$ defined in (19), it follows that

$$
\begin{array}{r}
\dot{\mathcal{V}}_{0}\left(\tau, \chi_{k}\right) \leq \frac{1}{T_{k}} \zeta_{k}(\tau)^{\prime}\left[\Pi_{10}+\tau \Pi_{2}+\left(T_{k}-2 \tau\right) \Pi_{3}\right. \\
\left.+\left(T_{k}-\tau\right) N R^{-1} N^{\prime}\right] \zeta_{k}(\tau),
\end{array}
$$

where the upper bound has been obtained by noting that, for any matrix $N$ in $\mathbb{R}^{\left(3 n_{p}+n_{c}+m\right) \times n_{p}}$ and any symmetric positive definite matrix $R \in \mathbb{R}^{n_{p} \times n_{p}}$, the following inequality holds [27]:

$$
\begin{aligned}
-\int_{\tau}^{T_{k}} \dot{\chi}_{p, k}(\theta)^{\prime} R \dot{\chi}_{p, k}(\theta) d \theta \leq & 2 \zeta_{k}(\tau)^{\prime} N\left(\chi_{p, k}(\tau)-\chi_{p, k}\left(T_{k}\right)\right) \\
& +\left(T_{k}-\tau\right) \zeta_{k}(\tau)^{\prime} N R^{-1} N^{\prime} \zeta_{k}(\tau) .
\end{aligned}
$$

Hence, combining the previous expressions, it follows that

$$
\begin{aligned}
\dot{\mathcal{W}}_{g}\left(\tau, \chi_{k}\right) \leq & \frac{1}{T_{k}} \zeta_{k}(\tau)^{\prime}\left[\Pi_{1}+\tau \Pi_{2}+T_{k} \Pi_{4}\right. \\
& \left.+\left(T_{k}-\tau\right) N R^{-1} N^{\prime}+\left(T_{k}-2 \tau\right) \Pi_{3}\right] \zeta_{k}(\tau),
\end{aligned}
$$

with $\Pi_{1}, \Pi_{2}, \Pi_{3}$ and $\Pi_{4}$ as defined in (19). A sufficient condition to ensure that $\dot{\mathcal{W}}_{g}<0$ consists in guaranteeing that

$$
\begin{aligned}
\Pi_{1}+\tau \Pi_{2}+ & \left(T_{k}-2 \tau\right) \Pi_{3}+T_{k} \Pi_{4} \\
& +\left(T_{k}-\tau\right) N R^{-1} N^{\prime}<0 .
\end{aligned}
$$

Note now that the inequality (27) is affine with respect to the variable $\tau$ in $\left[0, T_{k}\right]$. Then, by convexity, it suffices to ensure that (27) is negative for $\tau=0$ and $\tau=T_{k}$ (see [21] for more details). Thus $\dot{\mathcal{W}}_{g}<0$ if both inequalities

$$
\begin{aligned}
& \Pi_{1}+T_{k}\left(\Pi_{3}+\Pi_{4}+N R^{-1} N^{\prime}\right)<0, \\
& \Pi_{1}+T_{k}\left(\Pi_{2}-\Pi_{3}+\Pi_{4}\right)<0,
\end{aligned}
$$

are satisfied. Applying the same argument on $T_{k}$ for the interval $\left[\mathcal{T}_{1}, \mathcal{T}_{2}\right]$ and by using the Schur's complement, conditions $\Psi_{1}\left(\mathcal{T}_{i}\right)<$ 0 and $\Psi_{2}\left(\mathcal{T}_{i}\right)<0$, for $i=1,2$, given in (16) and (17), are obtained. Consider now matrix inequality (18). From Schur's complement, it follows that:

$$
\begin{aligned}
\chi_{k}(0)^{\prime} & P+V M_{f}+M_{f}^{\prime} V \\
& \left.-\left[\begin{array}{c}
0 \\
\left(K_{j}-G_{j}\right)^{\prime}
\end{array}\right] \alpha^{-1} u_{0 j}^{-2}\left[\begin{array}{c}
0 \\
\left(K_{j}-G_{j}\right)^{\prime}
\end{array}\right]^{\prime}\right) \chi_{k}(0)>0
\end{aligned}
$$

Since $\chi_{m, k}(0)=\chi_{p, k}(0)$, it follows that $M_{f} \chi_{k}(0)=0$. Thus, from (28), if $\chi_{k}(0) \in \mathcal{E}(P, \alpha)$, then $\bar{\chi}_{k}(0) \in \mathcal{S}$. Hence, provided (16) and (17) are verified, it follows that $\dot{\mathcal{W}}\left(\tau, \chi_{k}\right)<0$ and, from Lemma 1 , it follows that the state trajectory converges asymptotically to the origin.

Remark 2: Considering the particular choice of $G=K$, it follows that the sector condition (15) is globally valid, i.e. it is valid $\forall \bar{\chi}_{k}(0) \in$ $\mathbb{R}^{n_{p}+n_{c}}$. In this case, the satisfaction of conditions (16) and (17) ensures the global asymptotic stability of the origin of the closedloop system under the aperiodic sampling (see more details in [14]).

\section{OPTIMIZATION PROBLEMS}

In this section, we propose some optimization schemes based on the conditions of Theorem 1 to provide solutions to problems P1 and P2 stated in Section II.

\section{A. Optimization of the estimate of the domain of attraction (P1)}

Since the conditions of Theorem 1 guarantee that $\mathcal{E}(P, \alpha)$ is a RAS, an estimate of the actual region of attraction can be obtained by maximizing $\mathcal{E}(P, \alpha)$ considering some size criterion [29]. In this case, given $\mathcal{T}_{1}$ and $\mathcal{T}_{2}$, a solution to $\mathrm{P} 1$ can be obtained from the following optimization problem:

$$
\begin{gathered}
\min \operatorname{trace}(P)+\alpha \\
\text { subject to }(16),(17),(18)
\end{gathered}
$$

It should be noticed that relations (16) and (17) are nonlinear due to the product between variables $G$ and $U$. However, since $U$ is a diagonal matrix with dimension equal to the number of inputs, the solution to problem (29) can be computed by iteratively solving convex LMI problems over a grid on the elements of $U$. For instance, in the mono-input case $U$ reduces to a positive scalar and a line search algorithm can be applied. Another option may be to encapsulate the LMI optimization step in some overall nonlinear optimization procedure (such as the one implemented by fminsearch Matlab function). 


\section{B. Optimization of the allowable sampling interval (P2)}

The idea is to enlarge the interval $\left[\mathcal{T}_{1}, \mathcal{T}_{2}\right]$ for which regional stability is guaranteed for a given set of admissible initial conditions $\mathcal{X}_{0}$ under an aperiodic sampling policy with $T_{k} \in\left[\mathcal{T}_{1}, \mathcal{T}_{2}\right]$. In this case, assuming that the discrete-time controller (4) has been computed from a nominal periodic sampling period $\delta$, we can consider $\mathcal{T}_{1}=$ $\delta-\mu$ and $\mathcal{T}_{2}=\delta+\mu$, and the following optimization problem can be formulated:

$\max \mu$

$$
\text { subject to }(16),(17),(18), \mathcal{X}_{0} \subseteq \mathcal{E}(P, \alpha)
$$

The condition $\mathcal{X}_{0} \subseteq \mathcal{E}(P, \alpha)$ can be easily expressed as an LMI constraint if $\mathcal{X}_{0}$ is described either by an ellipsoid or by a polytope. Hence, this problem can be solved by iteratively increasing $\mu$ and testing LMI conditions.

\section{NumericAl EXAMPLE}

Let us consider the model of an unstable continuous-time balancing pointer system, expressed as in (1) with the following matrices:

$$
A_{p}=\left[\begin{array}{rr}
-0 & 1 \\
1 & 0
\end{array}\right], B_{p}=\left[\begin{array}{r}
0 \\
-1
\end{array}\right], C_{p}=\left[\begin{array}{ll}
1 & 1
\end{array}\right]
$$

with the saturation level $u_{0}=5$. Note that since $A_{p}$ is not Hurwitz, in view of the saturation constraint, only local asymptotic stability for the closed-loop system with discrete-time (or continuous-time) control can be guaranteed. We consider a stabilizing continuous-time dynamic controller (7), whose matrices are given by ([29], p.312):

$$
\bar{A}_{c}=0, \bar{B}_{c}=10, \bar{C}_{c}=1, \bar{D}_{c}=2, \bar{E}_{c}=0 .
$$

Next, we illustrate the application of our results under different perspectives of analysis.

\section{A. Influence of $\delta$}

Consider a discretized version of the controller obtained from (8). Let us first consider the influence of the discretization period $\delta$. With this aim, we apply the conditions of Theorem 1 considering a periodic sampling, i.e. $T_{k}=\delta=\mathcal{T}_{1}=\mathcal{T}_{2}, \forall k$. It follows that no solution for these conditions can be found for $\delta$ larger than $0.08 \mathrm{~s}$.

\section{B. Robustness with respect to aperiodic sampling}

Consider the discretized version of the controller obtained from (8) with $\delta=0.02 s$, which is given by:

$$
A_{c}=1 ; \quad B_{c}=0.2 ; \quad C_{c}=1 ; \quad D_{c}=2 .
$$

We evaluate now what happens under aperiodic sampling. For this we consider an allowable intersampling interval $T_{k} \in\left[\mathcal{T}_{1}, \mathcal{T}_{2}\right]$ centered at $\delta$, i.e. $\mathcal{T}_{1}=\delta-\mu$ and $\mathcal{T}_{2}=\delta+\mu$. Considering Problem $\mathrm{P} 1$, the influence of $\mu$ on the size of the obtained RAS is shown in Figure 1. Note that, as expected, the larger is $\mu$ (i.e. the larger is the admissible interval $\left.\left[\mathcal{T}_{1}, \mathcal{T}_{2}\right]\right)$, the smaller tends to be the region of stability $\mathcal{E}(P, \alpha)$. Moreover, the larger region is obtained with $\mu=0$, which corresponds to the periodic sampling case. Regarding Problem P2, conditions (16), (17), (18) become unfeasible when $\mu$ becomes larger than $\pm 24 \% \delta$.

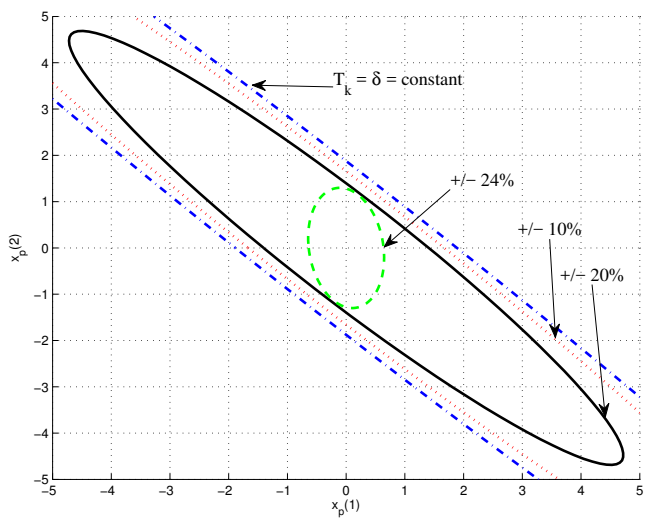

Fig. 1. Influence of the interval $\left[\mathcal{T}_{1}, \mathcal{T}_{2}\right]$ with $\mathcal{T}_{1}=\delta-\mu$ and $\mathcal{T}_{2}=\delta+\mu$ on the ellipsoidal regions of stability in the system plane $\left(x_{c}=0\right)$ considering $\delta=0.02$ and the following cases: $\mu=0$ (dashed-dotted line), $\mu=10 \% \delta$ (dotted line), $\mu=20 \% \delta$ (solid line) and $\mu=24 \% \delta$ (dashed green line).

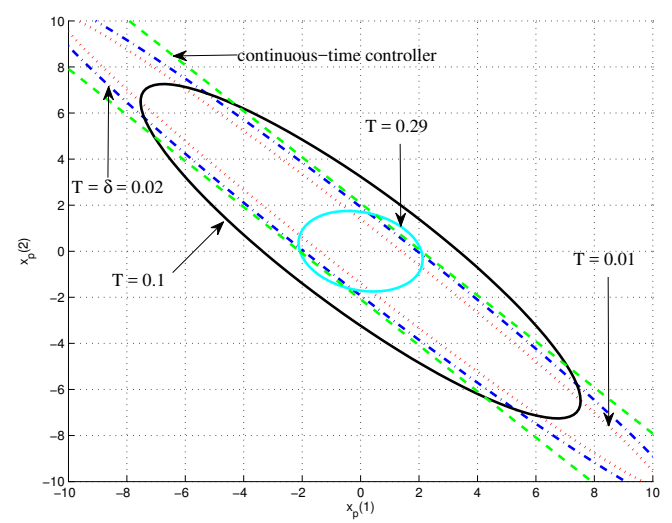

Fig. 2. Ellipsoidal regions of stability in the system plane $\left(x_{c}=0\right)$. Influence of a periodic sampling $T\left(\mathcal{T}_{1}=\mathcal{T}_{2}=T\right)$ when the controller is discretized with $\delta=0.02$. Cases with $T=\delta=0.02$ (dashed-dotted line), $T=0.01$ (dotted line), $T=0.1$ (solid line) and $T=0.29$ (solid line). The continuoustime case is plotted in dashed green line.

\section{Robust periodic implementation}

Consider now the dynamic discrete-time controller with the matrices given in (32), but that the actual periodic sampling period is different from $\delta=0.02 \mathrm{~s}$. In other words, we consider $\mathcal{T}_{1}=\mathcal{T}_{2}=$ $T \neq 0.02$. It can be observed that conditions (16), (17) and (18) remain feasible when reducing the sampling $T$ much below 0.02 , although the size of the stability domain is significantly degraded. Actually this domain shrinks as $T$ decreases from 0.02 . On the other hand, when $T$ is increased above the value of 0.02 , the problem remains feasible until $T=0.29$. This is illustrated in Figure 2 where it can be observed the influence of the sampling period $T$ on the size of the stability domain. For comparison purposes, the RAS obtained (from the application of Proposition 3.1 in [29]) considering the continuous-time implementation of the controller is also depicted in the figure.

An interesting aspect of this evaluation is that, with $T=\mathcal{T}_{1}=$ $\mathcal{T}_{2}$ larger than $\delta$, initial conditions not belonging to the region of attraction corresponding to the continuous-time closed-loop system (i.e. obtained from the connection between (1) and (7) with $\bar{u}(t)=$ $u(t)$ ) belong to the region of attraction obtained with the discretetime implementation of the controller. This is illustrated in Figure 3, 

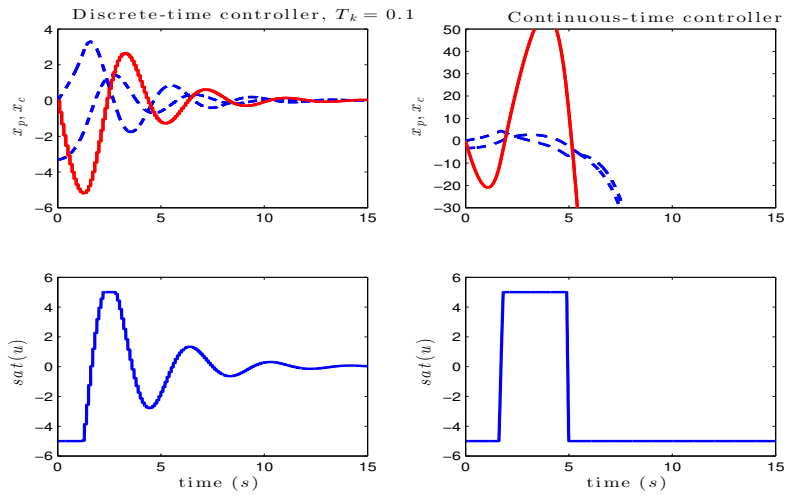

Fig. 3. Time response of the closed-loop system. Left-hand side: with discretized controller given by matrices in (32) and implemented with a periodic sampling $T=0.1$. Right-hand side: with the continuous-time controller given by matrices in (31).

which compares the time evolution of the closed-loop system with the discrete-time controller (32) implemented with $T=0.1$ and that one with the PI continuous-time controller (31), for the initial condition $x_{p}(0)=\left[\begin{array}{c}-3.3 \\ 0\end{array}\right]$ and $x_{c}(0)=0$. In fact, this initial condition belongs to the RAS obtained for the discrete-time controller, but does not belong to the one associated to the continuous-time implementation.

\section{CONCLUSION}

This paper provided theoretical conditions for the stability analysis of a closed-loop system composed of a linear continuous-time plant connected to a sampled-data dynamic output feedback controller through a saturating input. A hybrid model for the closed-loop system has been proposed. Then, considering a looped functional approach, conditions for assessing the regional asymptotic stability of the origin for the closed-loop system under aperiodic sampling have been derived in the form of matrix inequalities. From these conditions, LMI-based optimization problems have been proposed to compute estimates of the region of attraction of the origin for the hybrid nonlinear closed-loop system, as well as to maximize the interval of admissible inter sampling time for which the regional asymptotic stability of the closed-loop system can be ensured under aperiodic sampling.

The extension of the approach for the synthesis of stabilizing controllers taking into account the possibility of aperiodic sampling is an open issue. In this case, from the hybrid nature of the system, it is not possible to apply transformations like the ones proposed for instance in [25] and other works to convexify the problem. Hence, to find convex (or quasi-convex) conditions to address this problem can be seen as a challenge and is the subject of ongoing work.

\section{REFERENCES}

[1] K. J. Aström and B. Wittenmark. Computer Controlled Systems. Theory and Design. Information and System Sciences Series. Prentice-Hall, 1984.

[2] C. Briat. Convex conditions for robust stability analysis and stabilization of linear aperiodic impulsive and sampled-data systems under dwell-time constraints. Automatica, 49(11):3449-3457, 2013.

[3] C. Briat. Theoretical and numerical comparisons of looped functionals and clock-dependent Lyapunov functions -The case of periodic and pseudo-periodic systems with impulses. International Journal of Robust and Nonlinear Control, 2015.

[4] C. Briat and A. Seuret. A looped-functional approach for robust stability analysis of linear impulsive systems. Systems \& Control Letters, 61(10):980-988, 2012.
[5] T. Chen and B.A. Francis. Optimal sampled-data control systems. Springer-Verlag, 1995.

[6] S-T Chung and J.W. Grizzle. Sampled-data observer error linearization. Automatica, 26(6):997-1007, 1990.

[7] M.B.G. Cloosterman, L. Hetel, N. Van De Wouw, W.P.M.H. Heemels, J. Daafouz, and H. Nijmeijer. Controller synthesis for networked control systems. Automatica, 46(10):1584-1594, 2010.

[8] M.B.G. Cloosterman, N. Van De Wouw, W.P.M.H. Heemels, and H. Nijmeijer. Stability of networked control systems with uncertain timevarying delays. IEEE Trans. on Automatic Control, 54(7):1575-1580, 2009.

[9] D. Dai, T. Hu, A.R. Teel, and L. Zaccarian. Output feedback synthesis for sampled-data system with input saturation. In American Control Conference (ACC 2010), pages 1797-1802, Baltimore, USA, June 2010.

[10] E. Fridman. A refined input delay approach to sampled-data control. Automatica, 46(2):421-427, 2010.

[11] E. Fridman, A. Seuret, and J.-P. Richard. Robust sampled-data stabilization of linear systems: An input delay approach. Automatica, 40(8):1141-1446, 2004.

[12] H. Fujioka. Stability analysis of systems with aperiodic sample- andhold devices. Automatica, 45(3):771-775, 2009.

[13] R. Goebel, R.G. Sanfelice, and A.R. Teel. Hybrid Dynamical Systems Modeling, Stability and Robustness. Princeton University Press, 2012.

[14] J.M. Gomes da Silva Jr., I. Queinnec, A. Seuret, and S. Tarbouriech. Stability analysis of dynamic output controllers under aperiodic sampling and input saturation. In 21st International Symposium on Mathematical Theory of Networks and Systems (MTNS 2014), pages 1312-1317, 2014.

[15] J.M. Gomes da Silva Jr. and S. Tarbouriech. Anti-windup design with guaranteed region of stability: an LMI-based approach. IEEE Trans. on Automatic Control, 50(1):106-111, 2005.

[16] J.M. Gomes da Silva Jr. and S. Tarbouriech. Anti-windup design with guaranteed regions of stability for discrete-time linear systems. Systems \& Control Letters, 55(3):184-192, 2006.

[17] J.W. Grizzle and P.V. Kokotovic. Feedback linearization of sampled-data systems. IEEE Trans. on Automatic Control, 33(9):857-859, 1988.

[18] G. Herrmann, M.C. Turner, and I. Postlethwaite. Discrete-time and sampled-data anti-windup synthesis: stability and performance. International Journal of Systems Science, 37(2):91-113, 2006.

[19] T. Hu and Z. Lin. Control systems with actuator saturation: analysis and design. Birkhauser, Boston, 2001.

[20] C.-Y. Kao and H. Fujioka. On stability of systems with aperiodic sampling devices. IEEE Trans. on Automatic Control, 28(3):2085-2090, 2013.

[21] P. Naghshtabrizi, J.P. Hespanha, and A.R. Teel. Exponential stability of impulsive systems with application to uncertain sampled-data systems. Systems \& Control Letters, 57(5):378-385, 2008.

[22] D. Nešić and A.R. Teel. A framework for stabilization of nonlinear sampled-data systems based on their approximate discrete-time models. IEEE Trans. on Automatic Control, 49(7):1103-1122, 2004.

[23] D. Nešić, A.R. Teel, and E.D. Sontag. Formulas relating KL stability estimates of discrete-time and sampled-data nonlinear systems. Systems \& Control Letters, 38(1):49-60, 1999.

[24] Y. Oishi and H. Fujioka. Stability and stabilization of aperiodic sampleddata control systems using robust linear matrix inequalities. Automatica, 46(8):1327-1333, 2010.

[25] C. Scherer, P. Gahinet, and M. Chilali. Multiobjective output-feedback control via LMI optimization. IEEE Trans. on Automatic Control, 42(7):896-911, 1997.

[26] A. Seuret. A novel stability analysis of linear systems under asynchronous samplings. Automatica, 48(1):177-182, 2012.

[27] A. Seuret and J.M. Gomes da Silva Jr. Taking into account period variations and actuator saturation in sampled-data systems. Systems \& Control Letters, 61:1286-1293, 2012.

[28] N. Sivashankar and P.P. Khargonekar. Characterization of the $l_{2}$-induced norm for linear systems with jumps with applications to sampled-data systems. SIAM Journal on Control and Optimization, 32(4):1128-1150, 1994.

[29] S. Tarbouriech, G. Garcia, J.M. Gomes da Silva Jr., and I. Queinnec. Stability and Stabilization of Linear Systems with Saturating Actuators. Springer, 2011.

[30] Y. Yamamoto. New approach to sampled-data control systems - a function space method. In Proceedings of the 29th Conference on Decision and Control, pages 1882 - 1887, 1990.

[31] W. Zhang, M.S. Branicky, and S.M. Phillips. Stability of Networked Control Systems. IEEE Control Systems Magazine, 21(1):84-99, 2001. 